\title{
Chemoprevention of prostate cancer with nutrients and supplements
}

\author{
This article was published in the following Dove Press journal: \\ Cancer Management and Research \\ 5 April 20II \\ Number of times this article has been viewed
}

\author{
Hendrik Van Poppel' \\ Bertrand Tombal ${ }^{2}$ \\ 'Department of Urology, University \\ Hospital, KU Leuven, Leuven, Belgium; \\ ${ }^{2}$ Service d'Urologie, Cliniques \\ Universtaires Saint Luc, Brussels, \\ Belgium
}

\begin{abstract}
As the adult population is increasing, prostate cancer $(\mathrm{PCa})$ will become a considerable health problem in the next millennium. This has raised public interest in potential chemoprevention of this disease. As PCa is extremely common and generally slow to progress it is regarded as an ideal candidate for chemoprevention. At present, the 5 alpha-reductase inhibitors finasteride and dutasteride have been identified as preventive agents. This review describes whether selenium, alpha-tocopherol, isoflavones, lycopene green tea polyphenols, calcium, and resveratrol may be useful for decreasing the risk of $\mathrm{PCa}$ in men. Although encouraging results are present, some studies show negative results. Differences in study design, sample size, dose administered, and/or concentrations achieved in the body may be the reason for these inconsistencies. Today, chemopreventive agents may be appropriate for high-risk patients like those with high-grade prostatic intraepithelial neoplasia and other high-risk groups such as patients with elevated prostate specific antigen (PSA) and negative biopsy, rapid PSA velocity, and with a family history of PCa. Although larger randomized controlled studies are needed and epidemiologic evidence should be placed in a clinical context, physicians must be aware of these preventive opportunities in PCa care. Combinations of chemopreventive agents should be carefully investigated because mechanisms of action may be additive or synergistic.
\end{abstract}

Keywords: alpha-tocopherol, chemoprevention, isoflavones, lycopene, polyphenols, prostate cancer, selenium

\section{Introduction}

The incidence and mortality of prostate cancer $(\mathrm{PCa})$ shows strong variations worldwide with the highest rates in North America, Australia, Western and Northern Europe and the lowest rates in Japan and other Asian countries. Interestingly, however, the incidence of latent or clinical PCa in autopsy studies among men from Japan and the US is not substantially different. A diversity of genetic and environmental factors play a role in the development of PCa. In the last years, the risk for PCa has dramatically increased in Japan, possibly due to the introduction of the western diet. Moreover, migrant studies have shown an increase in PCa incidence in Asian men after emigration to the United States. ${ }^{1,2}$ These men adopt a western lifestyle with a high-fat, high-protein, low-fiber diet that lacks certain substances of the Asian diet such as plant-derived antioxidants, isoflavone-containing soy, and tea polyphenols that may protect against the development of cancer. ${ }^{3,4}$ Therefore, it is hypothesized that dietary changes and pharmacologic intervention could have an impact on $\mathrm{PCa}$ development and progression. ${ }^{5}$ Prostate cancer is an ideal target disease for chemoprevention because it is a very heterogeneous disease with a large subgroup of patients with nonaggressive disease. The situation
Correspondence: Hendrik Van Poppel Department of Urology, University Hospital, KU Leuven, B-3000 Leuven, Belgium

Tel +32 I 6346687

$\mathrm{Fax}+3216346931$

Email hendrik.vanpoppel@uz.kuleuven.ac.be 
has become critical with the rapid implementation of early detection tools, especially prostate specific antigen (PSA), so that most cancers are diagnosed years before they may become clinically evident and life-threatening for the patients. This poses a huge burden on the healthcare system because of the costs associated with its diagnosis and therapy. Therefore, even a moderate reduction or delay in the development of PCa accomplished through pharmacologic or dietary intervention could result in a considerable reduction in the incidence of PCa. PCa will become progressively important in patients over the age of 50 with increasing life expectancy in the next millennium. This suggests that $\mathrm{PCa}$ will become an even more considerable health problem and emphasizes the importance of preventive strategies. Chemoprevention is defined as the use of specific natural (dietary) or synthetic agents to prevent, delay, or slow the carcinogenic process. Primary chemoprevention should be a nontoxic and cost-effective intervention in a population of individuals at sufficiently increased risk. The simplest way for primary chemoprevention is the application of dietary nutrients or supplements in an easily available form, as most men prefer to take a tablet rather than to change their dietary habits.

Significant research has been carried out in chemoprevention for PCa over the past 2 years. Several randomized studies for prevention of PCa with pharmaceutical agents, dietary changes, and supplements have been published. The 5 alpha-reductase inhibitors finasteride and dutasteride have been identified as preventive agents and are discussed in many reviews. In the present review, we describe some of the more promising dietary nutrients and supplements and summarize the clinical studies on their effect on PCa, based on a review of the literature.

\section{Potential prostate cancer preventive agents Selenium and vitamin E}

Selenium is an essential trace element found in vegetables, grains, red meat, fish, poultry, and eggs. The concentration of selenium in the vegetables depends on how much of the mineral was in the soil where the plants grew. Selenium is distributed in body tissues and helps to make special proteins, called antioxidant enzymes, which play a role in preventing cell damage. Epidemiologic evidence provides support for a global cancer prevention effect. Vitamin $\mathrm{E}$ is an essential lipid-soluble antioxidant found in plant oils such as soy, corn, and olive oil. Other sources include nuts, seeds, and green leafy vegetables. It protects cells from free radicals. Several forms of vitamin $\mathrm{E}$ have been identified. The most active form with highest bioavailability in human tissues is alpha-tocopherol. The body is not capable of producing this substance, and it must be consumed in the diet or supplements for proper health.

The rationale for the use of selenium as a chemopreventive agent comes from the Nutritional Prevention of Cancer (NPC) Trial. On secondary analysis, this randomized, double-blind, placebo-controlled skin cancer prevention trial showed that it significantly reduced the overall incidence of PCa with a relative risk (RR) of $0.51(95 \%$ confidence interval [CI]: 0.29-0.87). The protective effect of selenium supplementation (200 $\mu \mathrm{g}$ daily) was restricted to those with lower baseline PSA ( $\leq 4 \mathrm{ng} / \mathrm{mL}$ ) (RR: 0.35 , 95\% CI: 0.13-0.87), although the interaction of baseline PSA and treatment was not statistically significant. Participants with baseline plasma selenium concentrations only in the lowest two tertiles $(<123.2 \mathrm{ng} / \mathrm{mL})$ had significant reductions in prostate cancer incidence. The unadjusted estimate showed a significant $65 \%$ reduction in $\mathrm{PCa}$ incidence with selenium supplementation. ${ }^{6}$ The rationale for the use of alphatocopherol as chemopreventive agent for PCa was based on the Alpha-Tocopherol, Beta-carotene Cancer Prevention (ATBC) study. ${ }^{7}$ On secondary analysis, the ATBC lung cancer prevention trial found a statistically significant 32\% reduction in PCa incidence (95\% CI: $12-47, P=0.002)$ in those receiving alpha-tocopherol $(50 \mathrm{mg} /$ day $)$. The reduction was evident in clinical PCa but not in latent cancer. In addition, a $41 \%$ reduction in PCa mortality (95\% CI: 1\%-65\%) was observed among men in the alpha-tocopherol group from 1985 to $1993 .{ }^{8}$ An additional follow-up of 12 years showed that higher serum alpha-tocopherol at baseline was associated with improved PCa survival (Hazard ratio [HR]: 0.67, 95\% CI: 0.45-1.00). The strongest survival relationship was seen for those who received alpha-tocopherol supplementation and were in the highest serum alpha-tocopherol quintile at baseline (HR: $0.51,95 \%$ CI: $0.20-0.90$ ) or at 3-year follow-up measurement (HR: 0.26, 95\% CI: 0.09-0.71). Neither serum nor supplemental beta-carotene or serum retinol had apparent effects on survival. ${ }^{9}$ These positive findings for alpha-tocopherol in the ATBC trial stand in contrast to those recently reported by the Physicians' Health Study II (PHS II) trial which evaluated much higher dosages of alpha-tocopherol for shorter periods.

Based on the indirect evidences, selenium and vitamin $\mathrm{E}$ were tested separately and in combination for the prevention of $\mathrm{PCa}$ in a randomized, prospective, double-blind, Phase III study, known as the Selenium and Vitamin E Cancer Prevention Trial (SELECT). The SELECT study was the 
largest cancer prevention study ever performed. It randomized 35,533 men to four groups: selenium $(200 \mu \mathrm{g} /$ day $)+$ placebo; vitamin E (400 IU/day) + placebo; selenium + vitamin E; or placebo + placebo. Eligibility criteria were age 50 years or older for African-Americans, 55 years or older for Caucasians, a serum PSA level of $4 \mathrm{ng} / \mathrm{mL}$ or less, a digital rectal examination not suspicious for cancer, and normal blood pressure. The primary endpoint was biopsy-confirmed PCa. No statistically significant differences in the rates of PCa were observed among the four groups. The HR was $1.13(99 \%$ CI: 0.95-1.35) in the vitamin E-alone group, 1.04 (99\% CI: $0.87-1.24)$ in the selenium-alone group and 1.05 (99\% CI: $0.88-1.25$ ) in the selenium with vitamin E group, compared with placebo. The study was terminated at 7 years (planned duration was 12 years) because no effect on the risk of PCa in these relatively healthy men could be demonstrated by neither selenium nor vitamin $\mathrm{E}$ or in combination at the doses and formulations used in the study. ${ }^{10}$ Concerns of the SELECT trial were a modest increase in the risk of $\mathrm{PCa}$ with vitamin $\mathrm{E}$ $(P=0.06)$ and in the risk of type 2 diabetes in the selenium group (RR: $1.07,95 \% \mathrm{CI}: 0.94-1.22, P=0.16){ }^{10}$

Reasons why selenium and/or vitamin E, alone or in combination, failed to prevent PCa in the SELECT trial are not clear. First, the high dose of vitamin E (400 IU/D of the alpha-tocopherol form) in SELECT may have been less effective than a lower dose such as the 8-fold lower $50 \mathrm{mg} / \mathrm{d}$ (roughly equivalent to $50 \mathrm{IU} / \mathrm{D}$ ) that produced the earlier positive secondary findings in the ATBC study. ${ }^{10}$ At a relatively high dose, natural vitamin $\mathrm{E}$ did not reduce PCa incidence. Achieving higher plasma or tissue levels of alpha-tocopherol within the physiologic range, such as through a $50 \mathrm{mg} / \mathrm{d}$ supplement, may have some prostate cancer (or other) preventive effect such as cell proliferation or tumor growth inhibition. This would also explain why in the PHS II study that enrolled 14,641 physicians aged 50 years or older including 1307 men with a history of prior cancer at randomization, no effect of high dose vitamin $\mathrm{E}$ (400 IU every other day; HR: 0.97, 95\% CI: 0.85-1.13) and similarly no effect of vitamin C (500 mg/day) (HR: 1.02, 95\% CI: 0.90-1.15) were found on PCa incidence during the 8 years follow-up. ${ }^{11}$ Second, several studies have suggested that vitamin $\mathrm{E}$ is more protective against $\mathrm{PCa}$ in smokers, and less than $60 \%$ of SELECT men were current or former smokers, whereas in the ATBC study all men were smokers. The fact that selenium was ineffective in preventing PCa could be due to the type of selenium used. In SELECT, $200 \mu \mathrm{g}$ of L-selenomethionine was chosen whereas in the NPC trial, the $200 \mu \mathrm{g}$ of high-selenium yeast contained only
$20 \%$ of L-selenomethionine. ${ }^{6,10}$ Therefore, future investigations are necessary to point out if selenium yeast is a better choice in the prevention of PCa. An alternative explanation is the existence of a genetic susceptibility to the effect of selenium.

For example, Chan et al have assessed manganese superoxide dismutase (SOD2) gene variants and plasma selenium in 489 patients with localized/locally advanced prostate cancer. SOD2 is an endogenous mitochondrial enzyme that metabolizes reactive oxygen species and superoxide anions to oxygen and hydrogen peroxide. Several polymorphisms of SOD2 have been identified, including, a single nucleotide permutation that encodes either an alanine (A) or a valine (V). SOD2 genotype alone was not associated with disease aggressiveness, whereas higher versus lower selenium levels were associated with a slightly increased likelihood of presenting with aggressive disease (RR: 1.35; 95\% CI: 0.99-1.84). There was evidence of an interaction between SOD2 and selenium levels such that among men with the AA genotype, higher selenium levels were associated with a reduced risk of presenting with aggressive disease (RR: 0.60; 95\% CI: 0.32-1.12), whereas among men with a V allele, higher selenium levels were associated with an increased risk of aggressive disease (for VV or VA men, RR: 1.82; 95\% CI: 1.27-2.61; $P$ for interaction $<0.007){ }^{12}$

Finally, it may be hypothesized that the positive effects of selenium in the NPC study and the positive effects of vitamin $\mathrm{E}$ on $\mathrm{PCa}$ incidence in the ATBC trial could have been due to chance in secondary analyses. In addition, recently published results from the Prostate Cancer Prevention Trial found no significant association between vitamin $\mathrm{E}$ and selenium and the incidence of $\mathrm{PCa} .{ }^{13}$ Moreover, longterm supplemental intake of vitamin $\mathrm{E}$ ( $\geq 400 \mathrm{IU} /$ day) in the VITamins And Lifestyle (VITAL) study was not associated with PCa risk overall; however, the risk of clinically relevant advanced disease was reduced with greater long-term (10-year average intake) vitamin E supplementation. ${ }^{14}$ Currently, several prevention studies are ongoing or have been completed. A Canadian randomized Phase III trial is investigating the effect of combining vitamin $\mathrm{E}$, selenium, and soy protein in preventing PCa in 306 patients who have high-grade prostatic intraepithelial neoplasia (HGPIN) (ClinicalTrials.gov Identifier NCT00064194). HGPIN is generally considered as a precursor of PCa. Prevention of $\mathrm{PCa}$ is desirable and men with HGPIN would be suitable, high-risk patients. A randomized, double-blind, placebocontrolled, Phase III trial (Southwest Oncology Group protocol 9917) funded by a National Cancer Institute program 
supporting pivotal prevention trials is ongoing to evaluate the effectiveness of selenium alone in preventing $\mathrm{PCa}$ in approximately 450 patients aged 40 years or older who have HGPIN and PSA levels of $\leq 10 \mathrm{ng} / \mathrm{mL}$ (ClinicalTrials.gov Identifier NCT00030901). ${ }^{15}$ Initial results were presented at the Annual Meeting of the American Urological Association in 2010. On average, 1.7 cores were involved with PIN on the first biopsy and 0.9 cores on the second biopsy. Treatment compliance was good with $80 \%$ of patients still taking the drug at the end of 3 years but $35 \%$ of patients were not accessible for the primary endpoint due to not getting the final prostate biopsy on time, or at all. At the time of this analysis, 50 patients on selenium and 50 patients on placebo had PCa. The tumors were mostly Gleason score $2-6$ with no difference between groups. The author concluded that $200 \mathrm{mg}$ of selenium does not prevent $\mathrm{PCa}$ in men with HGPIN. ${ }^{16}$

Further research is required to determine the choice of selenium type, before it can be recommended in the prevention of PCA. Caution is necessary when recommending high doses of selenium and/or vitamin $\mathrm{E}$ for the prevention of PCa.

\section{Isoflavones}

Isoflavones, a subclass of the flavonoids, are plant-derived compounds with weak estrogenic activity and therefore classified as phytoestrogens. Phytoestrogens have been suggested to have a preventive effect against various cancers. ${ }^{17}$ Soy foods are a rich source of isoflavones. Isoflavone intake in Asian countries is approximately $50 \mathrm{mg}$ daily, which is about ten times higher than intake in western countries. ${ }^{18}$ The main isoflavones found in most soy products are genistein, daidzein, and glycitein. In vitro genistein and daidzein inhibit the growth of PCa cells ${ }^{19,20}$ and cause apoptosis..$^{21,22}$ The mechanism of action of the isoflavones in soy products is not entirely clear. The biphasic bioactivity of genistein with low doses promoting cell growth and higher doses inhibiting growth requires caution in determining therapeutic doses of genistein alone or in combination with other therapies. Further investigation is warranted. ${ }^{23}$ In addition to epidemiologic data, experimental studies in vitro and in vivo have also demonstrated protective effects of isoflavones against PCa development. Among these effects, isoflavones possess weak estrogen activity, inhibit tyrosine protein kinases, block angiogenesis, and reduce serum testosterone levels. ${ }^{24,25}$ They also inhibit 5 alpha-reductase, an enzyme that metabolizes testosterone to dihydrotestosterone. ${ }^{26}$ Isoflavones are further metabolized in humans to many different intermediates, such as equol, perhaps the best-studied metabolite of daidzein.
Equol is ten times more potent than daidzein in retarding PCa growth. ${ }^{27}$

A cross-national study found that in the 42 countries for which there were appropriate data, soy products were significantly protective for $\mathrm{PCa} .{ }^{28}$ Several studies show that dose-dependent serum isoflavone levels are associated with a decreased risk for PCa. ${ }^{29-32}$ A case-control study including 200 patients and 200 age-matched controls evaluated the effects of isoflavones, fatty acids, vitamins, and minerals in the Japanese diet on PCa risk. They found that the isoflavones may be an effective dietary protective factor against PCa in Japanese men. The odds ratio for the highest quartile ( $\geq 89.9 \mathrm{mg} /$ day) compared with the lowest quartile $(<30.5 \mathrm{mg} /$ day $)$ of isoflavone intake was 0.42 (95\% CI: $0.24-0.72, P<0.01) .{ }^{31}$ Kurahashi et al (2007) investigated the association between isoflavone intake and risk of $\mathrm{PCa}$ in a prospective study of Japanese men ages 45 to 75 years who generally consume large amounts of soy. During follow-up from 1995-2004, 307 men were newly diagnosed with PCa, of which 74 cases were advanced, 220 cases were organ localized, and 13 cases were of an undetermined stage. Men with the highest intake of isoflavones (as genistein $\geq 32.8 \mathrm{mg} /$ day) had a decreased risk of $\mathrm{PCa}$ compared with those with the lowest intake of isoflavones (as genistein $<13.2 \mathrm{mg} /$ day). The authors found that isoflavone consumption was associated with a dose-dependent decrease in the risk of localized PCa, with relative risks for men aged $\geq 60$ years in the highest quartile of genistein, daidzein, and soy food consumption compared with the lowest of 0.52 (95\% CI: $0.30-0.90, P_{\text {trend }}=0.03$ ), 0.50 (95\% CI: $\left.0.28-0.88, P_{\text {trend }}=0.04\right)$, and $0.52(95 \% \mathrm{CI}$ : $\left.0.29-0.90, P_{\text {trend }}=0.01\right)$, respectively. In contrast, isoflavone consumption tended to be associated with an increased risk of advanced PCa. ${ }^{32}$ One mechanism of PCa risk reduction by isoflavones seems to involve estrogen receptor $\beta$ in prostate tissue. ${ }^{33}$ Cancer with higher metastatic potential is correlated with the complete or partial loss of the expression of estrogen receptor $\beta{ }^{34,35}$ Methodologic strengths of the study are the prospective design which reduces the probability of recall bias that is inherent to case-control studies, the fact that isoflavone intake was evaluated using a validated questionnaire and the large variation in isoflavone consumption..$^{32}$ Travis et al have examined plasma concentrations of phytoestrogens in relation to risk for subsequent $\mathrm{PCa}$ in a case-control study nested in the European Prospective Investigation into Cancer and Nutrition (EPIC). Higher plasma concentrations of genistein were associated with lower risk of PCa: RR among men in the highest vs the lowest fifth, 0.71 (95\% CI: $0.53-0.96$, $\left.P_{\text {trend }}=0.03\right)$. No statistically significant associations were 
observed for circulating concentrations of daidzein, equol, enterolactone, or enterodiol in relation to overall risk for prostate cancer. ${ }^{36}$

A nested case-control study conducted as part of the large Japan Public Health Center (JPHC)-based prospective cohort study investigated the effect of isoflavones as measured in plasma on subsequent PCa. This study is the first large study to investigate the association between plasma isoflavones and PCa to identify preventive effects of plasma isoflavones on localized PCa. In total, 20 newly diagnosed PCa were identified. Two matched controls for each case were selected from the cohort. They found that high plasma genistein (odds ratio (OR): $0.54,95 \% \mathrm{CI}: 0.29-1.01, P_{\text {trend }}=0.03$ for highest vs lowest group) and equol levels (OR: 0.43, 95\% CI: $0.22-0.82, P_{\text {trend }}=0.02$, for highest vs lowest group) were associated with a decreased risk of localized $\mathrm{PCa}$, with dose dependency. These findings suggest that plasma isoflavone levels may be protective against the development of localized PCa. Plasma isoflavone levels were not statistically significantly associated with the risk of advanced $\mathrm{PCa} .{ }^{37}$ A prospective study reported a decrease in PSA levels and lowered incidence of progression to $\mathrm{PCa}$ in men with isolated HGPIN in response to a 6-month supplementation with two tablets of Prevalon ${ }^{\circledR}$ (Madaus, Köln, Germany) per day. One tablet consists of $100 \mu \mathrm{g}$ selenium, $30 \mathrm{mg}$ vitamin E, and $50 \mathrm{mg}$ soy isoflavonoids (21 mg genistin, $17.6 \mathrm{mg}$ daidzin, and $11.4 \mathrm{mg}$ glycitin). The PCa risk throughout the study period was $25 \%$ in the group with a stable or declining PSA level from baseline and 52.2\% $(\mathrm{n}=48,67.6 \%)$ in the group with a rising PSA level from baseline $(n=23,32.4 \%)$ $(P=0.0458) .{ }^{38}$ Others have reported less PCa detection after 6 months of soy supplementation among high-risk patients. ${ }^{39}$ Other case-controlled studies support the idea of a protective effect of soy products for $\mathrm{PCa},{ }^{40,41}$ possibly because the prostatic isoflavone concentrations exceed the plasma levels after dietary supplementation. ${ }^{42-44}$ Levels of approximately $1 \mu \mathrm{g}$ isoflavone concentrations in the blood are regarded to be the highest achievable levels through intake of food. ${ }^{45}$ However, levels of isoflavone concentrations in prostatic tissue seem to be more relevant than blood levels. In a double-blind, randomized, placebo-controlled study, the administration of $82 \mathrm{mg}$ isoflavones per day $(27.2 \mathrm{mg} /$ tablet of total isoflavone, aglycone equivalent, contains $10.6 \mathrm{mg}$ genistein, $13.3 \mathrm{mg}$ daidzein, and $3.2 \mathrm{mg}$ glycitein) for 2 weeks in 19 men prior to radical prostatectomy (RP), resulted in a total isoflavone serum level of $0.7 \mu \mathrm{mol} / \mathrm{L}$ and a corresponding prostate tissue level of $2.3 \mu \mathrm{mol} / \mathrm{L}$. Thus, prostate tissue is able to accumulate isoflavones to potentially anticarcinogenic levels. ${ }^{41}$
Nevertheless, several studies have shown negligible benefits in men who received soy supplementation. ${ }^{46-48}$

Possible reasons for these inconsistent findings are differences in study design, sample size, doses administered, and/or isoflavone concentrations achieved in the body. ${ }^{49}$ The results of a very recent meta-analysis of the now available 14 epidemiological studies ( 8 on isoflavones) suggest that soy and isoflavone consumption is associated with a decreased risk of PCa. Soy food consumption was associated with a reduction in PCa risk of approximately $26 \%$ in men when the highest reported intake was compared with the lowest reported intake. The protective effect is related to the type and quantity of soy food consumed. The analysis on soy intake yielded a combined relative risk/odds ratio (RR/OR) of 0.74 (95\% CI: $0.63-0.89, P=0.01)$. The analysis of studies on nonfermented soy foods yielded a RR/OR of $0.70(95 \%$ CI: $0.56-0.88, P=0.01)$ and those on fermented soy foods yielded a combined RR/OR of 1.02 (95\% CI: 0.73-1.42; $P=0.92)$. The analysis of studies on isoflavones yielded a combined RR/OR of 0.88 (95\% CI: $0.76-1.02, P=0.09$ ). Further separate analyses showed a combined RR/OR of 0.52 (95\% CI: $0.34-0.81, P=0.01)$ from studies with Asian populations and 0.99 (95\% CI: $0.85-1.16, P=0.91)$ from studies with western populations. Well-designed studies on fermented and nonfermented soy foods in PCa etiology and prevention are required. ${ }^{50}$

To date, although there exists overwhelming data on epidemiologic studies, case control studies, and in vitro/vivo data that soy isoflavone may be a promising chemopreventive agent against PCA, there have been no published prospective randomized clinical studies with sufficient statistical power to assess whether isoflavone supplementation can reduce PCa development or delay PCa progression. Shortcomings for many studies published to date are small patient numbers, lack of randomization, short-term isoflavone administration, and possibly insufficient doses. ${ }^{51}$ Current ongoing clinical trials may help us understand the role of soy in the prevention of PCa.

\section{Lycopene}

Lycopene is a carotenoid without vitamin A activity that gives the red color to tomatoes and tomato-derived products. It is also available in other red fruits and vegetables such as red carrots, watermelons, pink grapefruit, and papayas. It possesses potent antioxidant activity and appears to have anticancer properties. ${ }^{52}$

An update of the results of the Health Professionals Follow-up Study, a prospective cohort study, revealed that 
frequent consumption of tomato products or lycopene is associated with a lower risk of PCa. The results were based on multiple dietary assessments using semiquantitative foodfrequency questionnaires from 1986 to 1998 and on 2481 cases of PCa. High lycopene intake was associated with a reduced risk of $\mathrm{PCa}$ ( $\mathrm{RR}$ for high vs low quintiles is 0.84 (95\% CI: $0.73-0.96 ; P_{\text {trend }}=0.003$ ). Intake of tomato sauce, the primary source of bioavailable lycopene, was associated with an even greater PCa risk reduction: RR for more than two servings/week vs less than one serving/month is 0.77 (95\% CI: $0.66-0.90, P_{\text {trend }}=<0.001$ ). These results suggest that lycopene may have a role in the prevention of $\mathrm{PCa} .{ }^{53}$ A large nested case-control study within the prospective Health Professionals Follow-up Study found a statistically significant inverse association between higher plasma lycopene concentrations and lower risk of $\mathrm{PCa}$, restricted to participants of 65 years or older (OR: $0.47,95 \%$ CI: $0.23-0.98$ ) at time of blood donation and those without a family history of PCa (OR: 0.43 , 95\% CI: 0.26-0.89). ${ }^{54}$ A Phase II clinical study showed that $30 \mathrm{mg}$ of oral lycopene supplementation for 3 weeks prior to RP reduced tumor size and plasma PSA level in localized PCa. Twelve of fifteen (84\%) men in the lycopene group and five of eleven $(45 \%)$ men in the control group had tumors $<4 \mathrm{~mL}$ in size $(P=0.22)$. Plasma PSA levels decreased by $18 \%$ in the intervention group, whereas they increased by $14 \%$ in the control group $(P=0.25) .{ }^{55}$ Chen et al $(2001)^{56}$ and Ansari and Gupta $(2003)^{57}$ also reported a decrease in PSA level with lycopene supplementation in patients with $\mathrm{PCa}$.

A meta-analysis of 11 case-control studies and 10 cohort studies or nested case-control studies showed that tomato products and lycopene may play a role in the prevention of $\mathrm{PCa}$ although the effect is modest and limited to high amounts of tomato products. The main findings were that, compared with nonfrequent users of tomato product (1st quartile of intake) the RR of PCa among consumers of high amounts of raw tomato (5th quintile of intake) was 0.89 (95\% CI: $0.80-1.00)$. For a high intake of cooked tomato products, the corresponding RR was 0.81 (95\% CI: 0.71-0.92). The RR of PCa related to an intake of one serving/day of raw tomato (200 g) was 0.97 (95\% CI: 0.85-1.10) for the case-control studies and 0.78 (95\% CI: 0.66-0.92) for cohort studies. For serum- or plasma-based studies, the corresponding RRs were 0.74 (95\% CI: 0.59-0.92) for all studies, $0.55(95 \%$ CI: 0.32-0.94) for case-control studies, and 0.78 (95\% CI: 0.61-1.00) for cohort studies. ${ }^{58}$ In a Phase II study, subjects with $\mathrm{PCa}$ were randomly assigned to receive a tomato capsule extract containing $15 \mathrm{mg}$ lycopene alone $(\mathrm{n}=38)$ or together with a capsule containing $40 \mathrm{mg}$ of a soy isoflavone mixture $(\mathrm{n}=33)$ twice daily orally for a maximum of 6 months. Thirty-five of 37 (95\%) evaluable patients in the lycopene group and 22 of 33 (67\%) evaluable patients in the lycopene plus soy isoflavone group achieved stable disease described as stabilization in serum PSA level. Future studies should further investigate the potential reactions between lycopene and soy isoflavones in PCa because both compounds are found in the diet and they are often taken together as supplements by PCa patients. ${ }^{48}$

Apparently lycopene is not only a promising component for chemoprevention of PCa, but can also play a possible beneficial role in patients diagnosed with benign prostate hyperplasia (BPH). In a pilot study, a total of 40 patients with histologically proven $\mathrm{BPH}$ were randomized to receive either lycopene (15 mg/day) or placebo for 6 months. The 6-month lycopene supplementation at a dose of $15 \mathrm{mg} /$ day decreased PSA levels in men $(P<0.05)$, inhibited disease progression, and improved the symptoms in $\mathrm{BPH}$ patients $(P<0.01)$ whereas there was no change in the placebo group. ${ }^{59}$ Indications for potential suppressive effects of lycopene on disease progression in BPH exist from a previous pilot study in $\mathrm{PCa}$ patients showing an increase in apoptotic cell death by lycopene in cancer-free BPH tissue. ${ }^{60}$ Moreover, analysis by experts of the World Cancer Research Fund shows that there is a sufficient body of evidence for the protective effect of lycopene-containing foods, especially tomatoes and their derivates, on PCa. This claim is based on five cohort studies and nine case control studies which investigated tomatoes; three cohort studies and 14 case-control studies investigated dietary lycopene; whilst six cohort studies and two case control studies investigated serum or plasma lycopene. Most of the studies decreased risk with increased intake (www.dietandcancerreport.org). ${ }^{61}$ A large nested case-control study in the prostate, lung, colorectal, and ovarian cancer screening study including $692 \mathrm{PCa}$ cases $^{62}$ and the recently published Prostate Cancer Prevention Trial ${ }^{13}$ including 9,559 participants found no correlation between lycopene and the incidence of PCa. Because chemoprevention is probably most effective in the early stages of $\mathrm{PCa}$, the next study carried out with lycopene in the treatment of HGPIN is worth mentioning. A randomized, double-blind, placebo-controlled, one-year study of lycopene $4 \mathrm{mg}$ twice a day has been conducted in 40 patients with HGPIN at transurethral resection of the prostate. PCa reduction after one year of treatment was $66 \%$. Lycopene was considered an effective chemopreventive agent in the treatment of HGPIN, with no toxicity and good tolerability ${ }^{63}$ 
Despite this randomized, double-blind study with lycopene, which proved to be an effective agent in the treatment of HGPIN, larger randomized controlled trials are required in well-defined patient sub-groups.

\section{Polyphenols}

Polyphenols are the largest group of constituents found in tea. Green tea contains catechins, a category of water-soluble polyphenolic substances. The four principal catechins are (-)-epicatechin (EC), (-)-epicatechin-3-gallate (ECG), (-)-epigallocatechin (EGC), and (-)-epigallocatechin-3-gallate (EGCG) ${ }^{64} \mathrm{EGCG}$, found in the highest concentration in green tea, is the most studied and most active of all green tea catechins (GTC) for the inhibition of oncogenesis and reduction of oxidative stress. The mode of action of polyphenols has not yet been fully determined. Several epidemiologic studies have focused on the lower incidence of $\mathrm{PCa}$ in Asian populations where green tea is consumed regularly as compared with western populations, suggesting that green tea is protective against $\mathrm{PCa}$.

Previous clinical investigations in healthy men have shown when a standardized green tea extract (eg, Polyphenon E) is used, the product is safe and tolerable. ${ }^{65}$ In 2006, a randomized, double-blind, placebo-controlled study was performed as a one-year proof-of-principle trial to assess the safety and efficacy of GTC capsules for the chemoprevention of PCa in HGPIN volunteers. ${ }^{66}$ Sixty patients were randomized to $600 \mathrm{mg}$ GTCs per day (ie, three $200 \mathrm{mg}$ capsules) or placebo. Each capsule contains 5.5\% EGC, 12.2\% EC, 51.9\% EGCG, $6.1 \%$ ECG, $75.7 \%$ total GTCs, and $<1 \%$ caffeine. After one year, only 1 of 30 GTC-treated men (incidence approximately 3\%) was found to have PCa compared to 9 of 30 placebo-treated men (incidence, 30\%). Altogether, these data suggest that a $90 \%$ reduction in developing PCa can be safely obtained by GTCs administration in men with HGPIN. This is the first study showing that GTCs have potent in vivo chemoprevention activity for human PCa. GTC treatment did not have a significant effect on PSA values throughout the study. In any case, the mean value of total PSA was always lower in patients randomized to GTCs than in patients on placebo. Secondary observations were changes in lower urinary tract symptoms (LUTS) as assessed by International Prostate Symptom Score (IPSS) and Quality of Life scores (QoL). IPSS and QoL scores of GTC-treated men with coexistent benign prostate hyperplasia (BPH) improved, reaching statistical significance in the case of IPSS. No significant side or adverse effects have been reported. ${ }^{66} \mathrm{~A}$ 2-year follow-up was performed in a subset of patients and showed that GTCs had a long-lasting effect on PCa prevention. ${ }^{67}$
A larger, randomized, double-blind, placebo-controlled study in 272 HGPIN patients in the United States will assess the rate of progression to $\mathrm{PCa}$ after treatment with either $200 \mathrm{mg}$ EGCG as Polyphenon E twice daily (ie, $400 \mathrm{mg}$ EGCG/day) or placebo over a 1 year period (ClinicalTrials. gov Identifier NCT00596011). A recently published, Phase II, open-label, single-arm, two-stage clinical trial determined the effects of short-term supplementation with the standardized green tea extract Polyphenon E on serum biomarkers in 26 patients with PCa. Daily doses of the standardized green tea extract administered during the interval between prostate biopsy and RP until time of RP contained $800 \mathrm{mg}$ of EGCG and lesser amounts of EC, EGC, and ECG (a total of $1.3 \mathrm{~g}$ of tea polyphenols). The results showed a significant reduction in serum levels of PSA, hepatocyte growth factor, and vascular endothelial growth factor in men with $\mathrm{PCa}$, with no elevation of liver enzymes. ${ }^{68}$ Results with green tea polyphenols for PCa chemoprevention are encouraging. Larger clinical trials of men at risk of $\mathrm{PCa}$ or with early stage $\mathrm{PCa}$ are needed to better assess the role of green tea polyphenols in the prevention of $\mathrm{PCa}$.

\section{Calcium}

Several large case-control and cohort studies have demonstrated that a high calcium intake from foods and/or supplements is associated with increased PCa risk. ${ }^{69-74}$ Giovannucci et al examined calcium intake in relation to prostate cancer risk using data from the Health Professionals Follow-up Study, a prospective cohort study of 47,750 male health professionals with no history of cancer other than nonmelanoma skin cancer at baseline. For high-grade $\mathrm{PCa}$ (Gleason score $\geq 7$ ), an association was observed for high versus low calcium intake (RR, 1.89 ; 95\% CI: 1.32-2.71; $\left.P_{\text {trend }}=0.005\right)$, but a nonsignificant, inverse association was observed for organ-confined, low-grade PCa (RR, 0.79; 95\% CI: 0.50-1.25; $\left.P_{\text {trend }}=0.09\right)$. Very high calcium intakes $(>1500 \mathrm{mg} /$ day) were associated with a higher risk of advanced and fatal PCa but not with well-differentiated, organ-confined cancers. ${ }^{72}$ Similarly, Allen et al have examined the consumption of calcium in relation to the risk of $\mathrm{PCa}$ among 142.251 men in the European Prospective Investigation into Cancer and Nutrition (EPIC) Study. After an average of 8.7 years of follow-up, there were 2727 incident cases of $\mathrm{PCa}$, of which 1131 were known to be localized and 541 advanced-stage disease. Total dietary calcium intake and calcium intake from dairy foods were associated with an increased risk of $\mathrm{PCa}$ (hazard ratio for the highest vs the lowest fifth of intake were 1.17, 95\% CI: $1.00-1.35$ 
for total dietary calcium and 1.18, 95\% CI: $1.03-1.36$ for dairy calcium). Calcium intake from nondairy foods was not associated with risk for PCa. ${ }^{71}$ This is in contrast with the finding of the Prostate Cancer Preventive Trial where calcium intake was positively associated with risk of lowgrade cancer but inversely associated with risk of highgrade cancer (for quartile 4 vs quartile 1, odds ratios were 1.27 (95\% CI: 1.02-1.57) and 0.43 (95\% CI: 0.21-0.89), respectively). No evidence has been found that very high dietary calcium intakes $(>1400 \mathrm{mg} /$ day $)$ were associated with increased risk of high-grade $\mathrm{PCa} .{ }^{13}$ This inconsistency in findings may require further investigation.

\section{Resveratrol}

Resveratrol (3,5,4'-trihydroxystilbene), a natural stilbenoid present in red wine, grapes, berries, peanuts, and dietary supplements as well as polyhydroxy analogs of resveratrol have potential cancer chemopreventive properties. It has sensitization/enhancing activities against tumor cells when used in combination with standard cancer chemotherapeutics. ${ }^{75}$ Recent findings strongly suggest that suppressor of cytokine signaling (SOCS-3), an antiapoptotic molecule that is upregulated in $\mathrm{PCa}$ is one of the proteins that influence the ability of resveratrol and tumor necrosis factor (TNF)-related apoptosis-inducing ligand (TRAIL) to cause programmed cell death in $\mathrm{PCa} .{ }^{76}$ Future studies should concentrate on the determination of molecular mechanisms of chemosensitization, efficacy of resveratrol combinations by clinically relevant in vivo studies, and demonstration of safety and effectiveness of combinations in humans. ${ }^{77}$

\section{Who should receive chemoprevention for $\mathrm{PCa}$ ?}

For high-risk patients, chemopreventive agents may be discussed, as the risk of PCa may justify the cost and potential side effects of these agents. It also seems reasonable to believe that chemoprevention strategies are more effective in high-risk groups. Nevertheless, the identification of high-risk groups is at this moment not easy. Patients with isolated HGPIN on prostate biopsies constitute a unique and well-demarcated risk group for PCa. Prognostic, randomized data on chemopreventive strategies in HGPIN are scarce, but seem promising. Other high-risk groups include those above 40 years of age, elevated PSA levels, rapid PSA velocity, sub-Saharan African ethnicity, a family history of PCa or with specific genes, or obese men with insulin resistance and those who would benefit from early diagnosis and treatment with at least $10-15$ years of life expectancy.

\section{Conclusions and future perspectives}

The concept of primary prevention is relatively new in the topic of prostate cancer and the effect of chemopreventive agents such as soy isoflavones, lycopene, and green tea polyphenols show encouraging results, although larger Phase III studies are needed. Some studies investigating selenium, alpha-tocopherol, and lycopene in the prevention of $\mathrm{PCa}$ show negative results. Differences in study design, sample size, dose administered, and/or concentrations achieved in the body may be the reason for these inconsistencies. The impact of calcium and resveratrol on $\mathrm{PCa}$ risk requires further investigation. Today, chemopreventive agents may be appropriate for high-risk patients such as patients with isolated HGPIN, elevated PSA, and negative biopsy, but are not appropriate for routine recommendation. Future research should focus on determining the target population for $\mathrm{PCa}$ chemoprevention. Large prospective randomized studies are required to define the benefits of chemopreventive agents for PCa. Some of these studies are ongoing and results are eagerly awaited. Combinations of chemopreventive agents for PCa should be carefully investigated because mechanisms of action may be additive or synergistic.

\section{Disclosure}

No conflicts of interest were declared in relation to this paper.

\section{References}

1. Shimizu H, Ross RK, Bernstein L, Yatani R, Henderson BE, Mack TM. Cancers of the prostate and breast among Japanese and white immigrants in Los Angeles County. Br J Cancer. 1991;63(6):963-966.

2. Marks LS, Kojima M, Demarzo A, et al. Prostate cancer in native Japanese and Japanese-American men: effects of dietary differences on prostatic tissue. Urology. 2004;64(4):765-771.

3. Lee J, Demissie K, Lu SE, Rhoads GG. Cancer incidence among Korean-American immigrants in the United States and native Koreans in South Korea. Cancer Control. 2007;14(1):78-85.

4. Chan JM, Stampfer MJ, Giovannucci EL. What causes prostate cancer? A brief summary of the epidemiology. Semin Cancer Biol. 1998;8(4):263-273.

5. Syed DN, Khan N, Afaq F, Mukhtar H. Chemoprevention of prostate cancer through dietary agents: progress and promise. Cancer Epidemiol Biomarkers Prev. 2007;16(11):2193-2203.

6. Duffield-Lillico AJ, Dalkin BL, Reid ME, et al. Selenium supplementation, baseline plasma selenium status and incidence of prostate cancer: an analysis of the complete treatment period of the Nutritional Prevention of Cancer Trial. BJU Int. 2003;91(7):608-612.

7. The Alpha-Tocopherol BCCPSG. The effect of vitamin E and beta carotene on the incidence of lung cancer and other cancers in male smokers. The Alpha-Tocopherol, Beta Carotene Cancer Prevention Study Group. N Engl J Med. 1994;330(15):1029-1035.

8. Heinonen OP, Albanes D, Virtamo J, et al. Prostate cancer and supplementation with alpha-tocopherol and beta-carotene: incidence and mortality in a controlled trial. J Natl Cancer Inst. 1998;90(6):440-446.

9. Watters JL, Gail MH, Weinstein SJ, Virtamo J, Albanes D. Associations between alpha-tocopherol, beta-carotene, and retinol and prostate cancer survival. Cancer Res. 2009;69(9):3833-3841. 
10. Lippman SM, Klein EA, Goodman PJ, et al. Effect of selenium and vitamin $\mathrm{E}$ on risk of prostate cancer and other cancers: the Selenium and Vitamin E Cancer Prevention Trial (SELECT). JAMA. 2009;301(1): 39-51.

11. Gaziano JM, Glynn RJ, Christen WG, et al. Vitamins E and C in the prevention of prostate and total cancer in men: the Physicians' Health Study II randomized controlled trial. JAMA. 2009;301(1):52-62.

12. Chan JM, Oh WK, Xie W, et al. Plasma selenium, manganese superoxide dismutase, and intermediate-or high-risk prostate cancer. J Clin Oncol. 2009;27(22):3577-3583.

13. Kristal AR, Arnold KB, Neuhouser ML, et al. Diet, supplement use, and prostate cancer risk: results from the prostate cancer prevention trial. Am J Epidemiol. 2010;172(5):566-577.

14. Peters U, Littman AJ, Kristal AR, Patterson RE, Potter JD, White E. Vitamin $\mathrm{E}$ and selenium supplementation and risk of prostate cancer in the Vitamins and lifestyle (VITAL) study cohort. Cancer Causes Control. 2008;19(1):75-87.

15. Marshall JR, Sakr W, Wood D, et al. Design and progress of a trial of selenium to prevent prostate cancer among men with high-grade prostatic intraepithelial neoplasia. Cancer Epidemiol Biomarkers Prev. 2006;15(8):1479-1484.

16. Marshall JR, Tangen CM, Sakr WA, et al. Southwest Oncology Group (SWOG) trial S9917: randomized phase 3 trial of selenium supplementation to prevent prostate cancer among men with high grade prostatic intraepithelial neoplasia (HGPIN). American Urological Association Annual Meeting, May 29-June 3, 2010, San Francisco, CA.

17. Adlercreutz H. Phyto-oestrogens and cancer. Lancet Oncol. 2002;3(6): 364-373.

18. Messina M, Nagata C, Wu AH. Estimated Asian adult soy protein and isoflavone intakes. Nutr Cancer. 2006;55(1):1-12.

19. Swami S, Krishnan AV, Peehl DM, Feldman D. Genistein potentiates the growth inhibitory effects of 1,25-dihydroxyvitamin D3 in DU145 human prostate cancer cells: role of the direct inhibition of CYP24 enzyme activity. Mol Cell Endocrinol. 2005;241(1-2):49-61.

20. Swami S, Krishnan AV, Moreno J, Bhattacharyya RB, Peehl DM, Feldman D. Calcitriol and genistein actions to inhibit the prostaglandin pathway: potential combination therapy to treat prostate cancer. J Nutr. 2007;137(1 Suppl):205S-210S.

21. Davis JN, Kucuk O, Sarkar FH. Expression of prostate-specific antigen is transcriptionally regulated by genistein in prostate cancer cells. Mol Carcinog. 2002;34(2):91-101.

22. Lakshman M, Xu L, Ananthanarayanan V, et al. Dietary genistein inhibits metastasis of human prostate cancer in mice. Cancer Res. 2008 68(6):2024-2032.

23. El Touny LH, Banerjee PP. Identification of a biphasic role for genistein in the regulation of prostate cancer growth and metastasis. Cancer Res. 2009;69(8):3695-3703.

24. Nagata $\mathrm{C}$, Inaba S, Kawakami N, Kakizoe T, Shimizu H. Inverse association of soy product intake with serum androgen and estrogen concentrations in Japanese men. Nutr Cancer. 2000;36(1):14-18.

25. Holzbeierlein JM, McIntosh J, Thrasher JB. The role of soy phytoestrogens in prostate cancer. Curr Opin Urol. 2005;15(1):17-22.

26. Evans BA, Griffiths K, Morton MS. Inhibition of 5 alpha-reductase in genital skin fibroblasts and prostate tissue by dietary lignans and isoflavonoids. J Endocrinol. 1995;147(2):295-302.

27. Hedlund TE, Johannes WU, Miller GJ. Soy isoflavonoid equol modulates the growth of benign and malignant prostatic epithelial cells in vitro. Prostate. 2003;54(1):68-78

28. Hebert JR, Hurley TG, Olendzki BC, Teas J, Ma Y, Hampl JS. Nutritional and socioeconomic factors in relation to prostate cancer mortality: a cross-national study. J Natl Cancer Inst. 1998;90(21):1637-1647.

29. Lee MM, Gomez SL, Chang JS, Wey M, Wang RT, Hsing AW. Soy and isoflavone consumption in relation to prostate cancer risk in China. Cancer Epidemiol Biomarkers Prev. 2003;12(7):665-668.

30. Ozasa K, Nakao M, Watanabe Y, et al. Serum phytoestrogens and prostate cancer risk in a nested case-control study among Japanese men. Cancer Sci. 2004;95(1):65-71.
31. NagataY, Sonoda T, Mori M, et al. Dietary isoflavones may protect against prostate cancer in Japanese men. J Nutr. 2007;137(8):1974-1979.

32. Kurahashi N, Iwasaki M, Sasazuki S, Otani T, Inoue M, Tsugane S. Soy product and isoflavone consumption in relation to prostate cancer in Japanese men. Cancer Epidemiol Biomarkers Prev. 2007;16(3): 538-545.

33. Ganry O. Phytoestrogens and prostate cancer risk. Prev Med. 2005; 41(1):1-6.

34. Horvath LG, Henshall SM, Lee CS, et al. Frequent loss of estrogen receptor-beta expression in prostate cancer. Cancer Res. 2001;61(14): 5331-5335.

35. Leav I, Lau KM, Adams JY, et al. Comparative studies of the estrogen receptors beta and alpha and the androgen receptor in normal human prostate glands, dysplasia, and in primary and metastatic carcinoma. Am J Pathol. 2001;159(1):79-92.

36. Travis RC, Spencer EA, Allen NE, et al. Plasma phyto-oestrogens and prostate cancer in the European Prospective Investigation into Cancer and Nutrition. Br J Cancer. 2009;100(11):1817-1823.

37. Kurahashi N, Iwasaki M, Inoue M, Sasazuki S, Tsugane S. Plasma isoflavones and subsequent risk of prostate cancer in a nested case-control study: the Japan Public Health Center. J Clin Oncol. 2008;26(36): 5923-5929.

38. Joniau S, Goeman L, Roskams T, Lerut E, Oyen R, Van Poppel H. Effect of nutritional supplement challenge in patients with isolated high-grade prostatic intraepithelial neoplasia. Urology. 2007;69(6):1102-1106.

39. Hamilton-Reeves JM, Rebello SA, Thomas W, Kurzer MS, Slaton JW. Effects of soy protein isolate consumption on prostate cancer biomarkers in men with HGPIN, ASAP, and low-grade prostate cancer. Nutr Cancer. 2008;60(1):7-13.

40. Jacobsen BK, Knutsen SF, Fraser GE. Does high soy milk intake reduce prostate cancer incidence? The Adventist Health Study (United States). Cancer Causes Control. 1998;9(6):553-557.

41. Heald CL, Ritchie MR, Bolton-Smith C, Morton MS, Alexander FE. Phyto-oestrogens and risk of prostate cancer in Scottish men. Br J Nutr. 2007;98(2):388-396.

42. Hedlund TE, Maroni PD, Ferucci PG, et al. Long-term dietary habits affect soy isoflavone metabolism and accumulation in prostatic fluid in caucasian men. J Nutr. 2005;135(6):1400-1406.

43. Rannikko A, Petas A, Rannikko S, Adlercreutz H. Plasma and prostate phytoestrogen concentrations in prostate cancer patients after oral phytoestogen supplementation. Prostate. 2006;66(1):82-87.

44. Gardner CD, Oelrich B, Liu JP, Feldman D, Franke AA, Brooks JD. Prostatic soy isoflavone concentrations exceed serum levels after dietary supplementation. Prostate. 2009;69(7):719-726.

45. Nielsen IL, Williamson G. Review of the factors affecting bioavailability of soy isoflavones in humans. Nutr Cancer. 2007;57(1):1-10.

46. Hussain M, Banerjee M, Sarkar FH, et al. Soy isoflavones in the treatment of prostate cancer. Nutr Cancer. 2003;47(2):111-117.

47. Spentzos D, Mantzoros C, Regan MM, et al. Minimal effect of a low-fat/ high soy diet for asymptomatic, hormonally naive prostate cancer patients. Clin Cancer Res. 2003;9(9):3282-3287.

48. Vaishampayan U, Hussain M, Banerjee M, et al. Lycopene and soy isoflavones in the treatment of prostate cancer. Nutr Cancer. 2007;59(1):1-7.

49. Perabo FG, Von Low EC, Ellinger J, von Rucker A, Muller SC, Bastian PJ. Soy isoflavone genistein in prevention and treatment of prostate cancer. Prostate Cancer Prostatic Dis. 2008;11(1):6-12.

50. Yan L, Spitznagel EL. Soy consumption and prostate cancer risk in men: a revisit of a meta-analysis. Am J Clin Nutr. 2009;89(4):1155-1163.

51. De Souza PL, Russell PJ, Kearsley JH, Howes LG. Clinical pharmacology of isoflavones and its relevance for potential prevention of prostate cancer. Nutr Rev. 2010;68(9):542-555.

52. Levy J, Bosin E, Feldman B, et al. Lycopene is a more potent inhibitor of human cancer cell proliferation than either alpha-carotene or beta-carotene. Nutr Cancer. 1995;24(3):257-266.

53. Giovannucci E, Rimm EB, Liu Y, Stampfer MJ, Willett WC. A prospective study of tomato products, lycopene, and prostate cancer risk. J Natl Cancer Inst. 2002;94(5):391-398. 
54. Wu K, Erdman JW Jr, Schwartz SJ, et al. Plasma and dietary carotenoids, and the risk of prostate cancer: a nested case-control study. Cancer Epidemiol Biomarkers Prev. 2004;13(2):260-269.

55. Kucuk O, Sarkar FH, Sakr W, et al. Phase II randomized clinical trial of lycopene supplementation before radical prostatectomy. Cancer Epidemiol Biomarkers Prev. 2001;10(8):861-868.

56. Chen L, Stacewicz-Sapuntzakis M, Duncan C, et al. Oxidative DNA damage in prostate cancer patients consuming tomato sauce-based entrees as a whole-food intervention. J Natl Cancer Inst. 2001;93(24): 1872-1879.

57. Ansari MS, Gupta NP. A comparison of lycopene and orchidectomy vs orchidectomy alone in the management of advanced prostate cancer. BJU Int. 2003;92(4):375-378; discussion 378.

58. Etminan M, Takkouche B, Caamano-Isorna F. The role of tomato products and lycopene in the prevention of prostate cancer: a metaanalysis of observational studies. Cancer Epidemiol Biomarkers Prev. 2004; 13(3):340-345.

59. Schwarz S, Obermuller-Jevic UC, Hellmis E, Koch W, Jacobi G, Biesalski HK. Lycopene inhibits disease progression in patients with benign prostate hyperplasia. J Nutr. 2008;138(1):49-53.

60. Kim HS, Bowen P, Chen L, et al. Effects of tomato sauce consumption on apoptotic cell death in prostate benign hyperplasia and carcinoma. Nutr Cancer. 2003;47(1):40-47.

61. World Cancer Research Fund/American Institute for Cancer Research. Food, nutrition, physical activity and the prevention of cancer. London, UK: WCRF International, 2007.

62. Peters U, Leitzmann MF, Chatterjee N, et al. Serum lycopene, other carotenoids, and prostate cancer risk: a nested case-control study in the prostate, lung, colorectal, and ovarian cancer screening trial. Cancer Epidemiol Biomarkers Prev. 2007;16(5):962-968.

63. Mohanty NK, Saxena S, Singh UP, Goyal NK, Arora RP. Lycopene as a chemopreventive agent in the treatment of high-grade prostate intraepithelial neoplasia. Urol Oncol. 2005;23(6):383-385.

64. Balentine DA, Wiseman SA, Bouwens LC. The chemistry of tea flavonoids. Crit Rev Food Sci Nutr. 1997;37(8):693-704.

65. Chow HH, Cai Y, Hakim IA, et al. Pharmacokinetics and safety of green tea polyphenols after multiple-dose administration of epigallocatechin gallate and polyphenon $\mathrm{E}$ in healthy individuals. Clin Cancer Res. 2003;9(9):3312-3319.

66. Bettuzzi S, Brausi M, Rizzi F, Castagnetti G, Peracchia G, Corti A. Chemoprevention of human prostate cancer by oral administration of green tea catechins in volunteers with high-grade prostate intraepithelial neoplasia: a preliminary report from a one-year proof-of-principle study. Cancer Res. 2006;66(2):1234-1240.
67. Brausi M, Rizzi F, Bettuzzi S. Chemoprevention of human prostate cancer by green tea catechins: two years later. A follow-up update. Eur Urol. 2008;54(2):472-473.

68. McLarty J, Bigelow RL, Smith M, Elmajian D, Ankem M, Cardelli JA. Tea polyphenols decrease serum levels of prostate-specific antigen, hepatocyte growth factor, and vascular endothelial growth factor in prostate cancer patients and inhibit production of hepatocyte growth factor and vascular endothelial growth factor in vitro. Cancer Prev Res (Phila). 2009;2(7):673-682.

69. Gao X, LaValley MP, Tucker KL. Prospective studies of dairy product and calcium intakes and prostate cancer risk: a meta-analysis. J Natl Cancer Inst. 2005;97(23):1768-1777.

70. Kesse E, Bertrais S, Astorg P, et al. Dairy products, calcium and phosphorus intake, and the risk of prostate cancer: results of the French prospective SU.VI.MAX (Supplementation en Vitamines et Mineraux Antioxydants) study. Br J Nutr. 2006;95(3):539-545.

71. Allen NE, Key TJ, Appleby PN, et al. Animal foods, protein, calcium and prostate cancer risk: the European Prospective Investigation into Cancer and Nutrition. Br J Cancer. 2008;98(9):1574-1581.

72. Giovannucci E, Liu Y, Stampfer MJ, Willett WC. A prospective study of calcium intake and incident and fatal prostate cancer. Cancer Epidemiol Biomarkers Prev. 2006;15(2):203-210.

73. Ahn J, Albanes D, Peters U, et al. Dairy products, calcium intake, and risk of prostate cancer in the prostate, lung, colorectal, and ovarian cancer screening trial. Cancer Epidemiol Biomarkers Prev. 2007;16(12): 2623-2630.

74. Mitrou PN, Albanes D, Weinstein SJ, et al. A prospective study of dietary calcium, dairy products and prostate cancer risk (Finland). Int J Cancer. 2007;120(11):2466-2473.

75. Hsieh TC, Wu JM. Resveratrol: biological and pharmaceutical properties as anticancer molecule. Biofactors. 2010;36(5):360-369.

76. Horndasch M, Culig Z. SOCS-3 antagonizes pro-apoptotic effects of TRAIL and resveratrol in prostate cancer cells. Prostate. February 9, 2011. [Epub ahead of print].

77. Gupta SC, Kannappan R, Reuter S, Kim JH, Aggarwal BB. Chemosensitization of tumors by resveratrol. Ann N Y Acad Sci. 2011;1215(1):150-160.
Cancer Management and Research

\section{Publish your work in this journal}

Cancer Management and Research is an international, peer-reviewed open access journal focusing on cancer research and the optimal use of preventative and integrated treatment interventions to achieve improved outcomes, enhanced survival and quality of life for the cancer patient. The journal welcomes original research, clinical \& epidemiological

\section{Dovepress}

studies, reviews \& evaluations, guidelines, expert opinion \& commentary, case reports \& extended reports. The manuscript management system is completely online and includes a very quick and fair peerreview system, which is all easy to use. Visit http://www.dovepress.com/ testimonials.php to read real quotes from published authors. 